\title{
Neuroendocrinology 1975;17:375-377
}

\section{Subject Index}

\section{Acetylcholine 1 ACTH 258}

costerone 225

- rhythm 203 Adrenalectomy 258 Adrenergic nerves 40 Amygdala 139 Anestrus 289 Anosmia 340, 362 Antagonism, serotonin 245 Anterior median eminence 75

- pituitary gland 92, 236 Antidiuretic hormone 139

- substance 139 Antiserum to LH-RH 12 Area, hypophysiotrophic 18 Arginine vasopressin 354

- vasotocin 354

Behavior, lordosis 309 Biological rhythms 48 Blinding 340, 362 Brattleboro rat 354

Castration 189 Cell proliferation 92 Cells, nerve 330 Circadian periodicity 62

Conscious dogs 75 Constant estrus 289

- light 167

Copulation 193

Cornification, vaginal 83

Corticosteroid 62

Corticosterone $125,154,203$

-, adrenal 225

Corticotrophin releasing hormone $1 \mathrm{CRF}$ production 18

Cycle, estrous 167 Cyclic mice 83 Cyproheptadine 245

Daily rhythms 319

Dense-core vesicles 211

Dexamethasone 203

5,6-Dihydroxytryptamine 62

3,4-Dimethoxyphenylethylamine 245

Diurnal rhythm 296

- rhythms 125 L-Dopa 147

- and aging 289 Dopamine 125

Effect of serotonin and melatonin 265 Ejaculation 309 Electron microscopy 40 Eminence, anterior median 75 -, median 330 Endocrine cells 40 Subject Index

Estrous cycle 167 Estrus, constant 289 Ether 258

Factor, luteinizing hormone-releasing 309

- , prolactin release-inhibiting 362

, prolactin-releasing 362

Fetus 225

Fluorescence microscopy 40

Gland, anterior pituitary 92,236

- , interstitial 40

-, pineal 362

, pituitary 12,139

Gonadotropic control 175

Gonadotropins 48, 309

Growth hormone 125, 245

Heifers 54

Hormone, antidiuretic 139

-, corticotrophin releasing 1

-, growth 125,245

-, luteinizing 362

-, - hormone-releasing 362

-, melanocyte-stimulating 296

Hormones, ovarian 309

1ljS-Hydroxyandrostenedione 27

5-Hydroxy-L-tryptophan 245

$17 \alpha$-Hydroxyprogesterone 27

6-Hydroxytryptamine 62

Hypophysectomy 75

Hypophysiotrophic area 18

Hypothalamic lesions 75

Hypothalamo-hypophyseal-gonadotrophin

release 265 Hypothalamo-hypophyseal system 225 Hypothalamus 1,12,175,211,236,283,354 -, medial basal 362 Hypothyroidism 147 
Immature rats 274 Interstitial gland 40

Laparotomy 211 Lateral ventricle 154 Lesions 193 -, hypothalamic 75 
-, radio-frequency 75 LH 236

- release 12, 283 LH-RF 189 LH-RH 12, 48, 274 Lordosis behavior 309 LRF release 283 Luteinizing hormone 362

- hormone-releasing factor 309

hormone 362

Medial basal hypothalamus 362 Median eminence 330 Melanocyte-stimulating hormone 296 Melatonin 245, 296 5-Methoxytryptamine 245 Microscopy, electron 40 -, fluorescence 40 Monoamines 147 Multiple unit activity 175

Neonatal 62 Nerve cells 330 Nerves, adrenergic 40 Neurohypophysis 354 Neurosecretion 211 Noradrenaline 1 Norepinephrine 62, 125, 154

17-OHCS secretion 75 Ovarian hormones 309 Ovariectomy 83, 211 Ovary 40 Ovulation 27, 83, 265, 274

Patterns of reproduction 289 Pentobarbital 285 Periodicity, circadian 62 Phenoxybenzamine 154 Pilocarpine 75 Pineal 296, 354

- gland 362 Pinealectomy 167, 340 Pituitary 48, 296

- gland 12, 139

- LH 83

- stalk 139

Subject Index

- transplant 18

Vasotocin,

arginine

Progesterone 236, 319

Vesicles,

dense-core

Prolactin 54, 245, 340, 362

- release-inhibiting factor 362

Prolactin-releasing factor 362

Proliferation, cell 92

Prostaglandin $\mathrm{E}_{2} 12,283$

Pseudopregnancies, repeat 289

Puberty 203, 319

Quail 175

Radio-frequency lesions 75

Radioimmunoassay 354

Rat 225

Release, hypothalamo-hypophyseal-

gonadotrophin 265 Repeat pseudopregnancies 289 Reproduction and aging 289 Reserpine 154 Rhythm, adrenal 203 -, diurnal 296 Rhythms, biological 48 -, diurnal 125

Serotonin 62, 125, 245

- antagonism 245

Sham ovariectomy 83

Sheep 265

Site of action of serotonin and melatonin

265 Stimulation of teats 54 Stress 154, 203, 225, 258 Suprachiasmatic nucleus 211 System, hypothalamo-hypophyseal 225

Testosterone 27 Transplant, pituitary 18 TRH 92 TSH 147

Ultrastructure 330 Uterine weight 83

Vaginal cornification 83 Vasopressin, arginine 354 